\title{
Down-regulation of P-glycoprotein expression contributes to an increase in Danshensu accumulation in the cerebral ischemia/reperfusion brain
}

\author{
YATING CHONG ${ }^{*}$, TIAN WANG ${ }^{*}$, WENYAN WANG ${ }^{*}$, LEIMING ZHANG, CHONG LI, \\ PENGFEI YU, HONGSHENG WANG and FENGHUA FU
}

Department of Pharmacology, School of Pharmacy, Yantai University, Yantai, Shandong 264005, P.R. China

Received August 16, 2011; Accepted November 21, 2011

DOI: $10.3892 / \mathrm{mmr} .2011 .691$

\begin{abstract}
P-glycoprotein (P-gp) plays a key role in the distribution and elimination of many clinically important therapeutic substances. P-gp inhibition is capable of increasing the concentration of various drugs in the brain. Danshensu is a main hydrophilic constituent of Salvia miltiorrhiza Bunge which is used traditionally to treat cerebrovascular diseases. In the present study, the effects of cerebral ischemia/reperfusion on the transport of Danshensu in the brain were observed by assaying the concentration at 15,30 and 60 min after cerebral ischemia/reperfusion in the brain of rats. Furthermore, the expression of P-gp in the brain was also measured. Results showed that Danshensu concentrations in the ischemic hemisphere significantly increased compared to those in the non-ischemic hemisphere and the brain of the sham group. P-gp expression was inhibited by cerebral ischemia/reperfusion to some extent, and down-regulated much more by Danshensu treatment. The findings indicate that the down-regulation of $\mathrm{P}$-gp expression induced by both cerebral ischemia/reperfusion and Danshensu treatment contributes to the increase of Danshensu accumulation in the brain.
\end{abstract}

\section{Introduction}

The blood-brain barrier (BBB) consists of two components. One forms a structural/physical barrier comprising the endothelial cells themselves and the extremely tight intercellular junctional complexes. The other forms a selective/biochemical barrier which includes a group of specific transport proteins $(1,2)$. P-glycoprotein (P-gp) is present in brain capil-

Correspondence to: Professor Fenghua Fu, Department of Pharmacology, School of Pharmacy, Yantai University, Yantai, Shandong 264005, P.R. China

E-mail: fufenghua@sohu.com

*Contributed equally

Key words: Danshensu, P-glycoprotein, blood-brain barrier, cerebral ischemia/reperfusion, central nervous system laries that form the $\mathrm{BBB}$, localized primarily to the luminal surface of the endothelial lining. P-gp appears to play a key role in restricting the entry of lipophilic drugs to the brain. It may also be responsible for removing certain substances from the brain to the blood (3). P-gp is considered to be the major key to central nervous system (CNS) entry of therapeutic drugs.

As P-gp greatly influences the therapeutic effects of CNS drugs, it is important to know the extent to which P-gp is pathophysiologically modulated. Currently, emerging evidence indicates that P-gp expression is altered during diseases, e.g., in epilepsy or stroke. Felix and Barrand reported that P-gp expression in brain capillary endothelial cells increased after being exposed to hydrogen peroxide over a period of 1 to 2 days (4). However, what happens to P-gp expression after stroke in vivo is largely uncertain. A previous study using a rat brain focal ischemia model found a loss of P-gp expression in the ischemic lesion followed by recovery of expression during the subsequent post-ischemic period when angiogenesis was occurring (5). One recent study found up-regulation of endothelial cell P-gp expression 3 to $24 \mathrm{~h}$ after focal cerebral ischemia in a mouse model (6). In contrast, Dazert and colleagues found there was no change in P-gp expression in the peri-infarct region of rats after middle cerebral artery occlusion (MCAO) (7).

Salvia miltiorrhiza Bunge has been widely used in China, Korea, Japan and other Asian countries in the treatment of cerebrovascular diseases. A recent study demonstrated that the extract of Salvia miltiorrhiza Bunge reduces the risk of ischemic stroke or a transient ischemic attack recurrence, and the reduction of risk was associated with its anti-inflammatory effects (8). It was also reported that Danshensu, the major hydrophilic constituent of Salvia miltiorrhiza Bunge, ameliorated the neurological deficit, the pathological change in brain tissue and decreased the percentage of neural cell apoptosis in cerebral ischemia/reperfusion rats (9). The results from our laboratory showed that Danshensu concentrations in the brain of rats pretreated with verapamil (an inhibitor of P-gp) significantly increased (10). However, to date, it remains unclear whether Danshensu penetrates across the BBB and whether P-gp has an effect on the Danshensu concentration in ischemic brain tissue after cerebral ischemia/reperfusion. In the present study, 
the effects of cerebral ischemia/reperfusion on the transport of Danshensu in the brain were observed. Expression of P-gp in cerebral ischemia/reperfusion and Danshensu treatment was also measured.

\section{Materials and methods}

Animals. Male Sprague-Dawley rats weighing 260-300 g were purchased from the Experimental Animal Center of Shandong Engineering Research Center for Natural Drugs (Yantai, China). The rats were housed in conventional cages with free access to food and water at a temperature of $22.0 \pm 2.0^{\circ} \mathrm{C}$ and a relative humidity of $50 \pm 10 \%$ with a 12 -h light/dark cycle. The present study was conducted in accordance with the Guiding Principles for the Care and Use of Laboratory Animals of Yantai University.

Drugs and chemicals. Danshensu (purity, 99.6\%) was obtained from Shandong Luye Pharmaceutical Co., Ltd. (Yantai, China) and ethyl acetate (analytical reagent) was purchased from Sinopharm Chemical Reagent Co., Ltd. (Shanghai, China). Anti-P-gp antibody was obtained from Thermo Fisher Scientific. Enhanced chemiluminescence (ECL) detection reagents and bicinchoninic acid (BCA) protein assay kit were obtained from Beyotime Institute of Biotechnology (Haimen, China).

Focal cerebral ischemia/reperfusion model. Cerebral ischemia was induced by an intraluminal MCAO technique as described by Longa et al (11). During surgical and treatment periods, the room temperature was maintained in the range of $25-28^{\circ} \mathrm{C}$, and rat rectal temperature was maintained at $37 \pm 0.5^{\circ} \mathrm{C}$ by a heat lamp. Briefly, rats were anesthetized with $10 \%$ chloral hydrate $(300 \mathrm{mg} / \mathrm{kg}$, i.p.). Following the skin incision, the left common carotid artery (CCA), the external carotid artery (ECA) and the internal carotid artery (ICA) were carefully exposed. A coated 6-0 filament with a rounded tip was introduced into an arteriotomy hole, fed distally into the ICA and advanced $11 \mathrm{~mm}$ from the carotid bifurcation. The ICA clamp was removed and focal cerebral ischemia started. After $2 \mathrm{~h}$ of MCAO, reperfusion was induced by withdrawing the filament. The sham operation group underwent the same procedure, however without vascular occlusion.

Experimental design. Eighty-one male Sprague Dawley rats were randomly assigned to 3 groups $(n=27)$ : the sham operation (sham), the cerebral ischemia/reperfusion (I/R) and the cerebral ischemia/reperfusion + Danshensu group (I/R + Danshensu). Before operation, 3 animals in each group were sacrificed by euthanasia and perfused with $100 \mathrm{ml}$ of ice-cold normal saline each. The left hemispheres were then removed for Western blot analysis. Immediately after reperfusion, the sham and I/R + Danshensu groups were treated intravenously with Danshensu $(15 \mathrm{mg} / \mathrm{kg})$ by tail vein and the rats in I/R group were treated with the same volume of normal saline. The rats $(n=8)$ in each subgroup were anesthetized with chloral hydrate $(300 \mathrm{mg} / \mathrm{kg}$, i.p.), and $5 \mathrm{ml}$ heparinized blood (1\% heparin, $100 \mu \mathrm{l}$ ) was collected from the abdominal aorta at 15, 30 and $60 \mathrm{~min}$ after reperfusion. The blood samples were centrifuged $\left(2500 \times \mathrm{g}, 4^{\circ} \mathrm{C}\right)$ for $10 \mathrm{~min}$ and plasma was separated. Moreover, the animals were perfused with $100 \mathrm{ml}$ of ice-cold normal saline. The brains were rapidly removed from the cranium, and the ischemic and non-ischemic hemispheres were separated and weighed. Then the ischemic and non-ischemic hemispheres were homogenized in $0.1 \mathrm{M}$ iced phosphate buffer ( $\mathrm{pH} 7.4$ ) to obtain $20 \%$ homogenates. A total amount of $3 \mathrm{ml}$ of ethyl acetate was added into $200 \mu \mathrm{l}$ of the homogenate, vortexed for $3 \mathrm{~min}$ and centrifuged $\left(2500 \mathrm{x} \mathrm{g}, 4^{\circ} \mathrm{C}\right)$ for $5 \mathrm{~min}$. The supernatants were evaporated to dryness under a gentle nitrogen stream at $40^{\circ} \mathrm{C}$. The levels of Danshensu in plasma and brain were detected by high performance liquid chromatography-electrospray ionization tandem mass spectrometry (LC-ESI-MS/MS). The P-gp expression in ischemic hemispheres was assayed by Western blot analysis.

Western blot analysis to determine the P-gp expression in rat brain. The brain tissues were lysed in RIPA buffer (Beyotime, Haimen, China) plus 1:100 volume of phenylmethylsulfonyl fluoride (PMSF). After centrifugation for $15 \mathrm{~min}(12000 \mathrm{x} \mathrm{g}$, $\left.4^{\circ} \mathrm{C}\right)$, the supernatant was obtained as membrane fractions for Western blotting. The protein concentration was determined by using a bicinchoninic acid (BCA) protein assay kit. Each sample was denatured at $100^{\circ} \mathrm{C}$ for $5 \mathrm{~min}$ mixed with 4X sodium dodecyl sulphate sample buffer (Invitrogen, Carlsbad, CA). Equivalent amounts of proteins (50 $\mu \mathrm{g})$ were loaded on Tris-glycine, SDS-polyacrylamide gels for fractionation at $90 \mathrm{~V}$ for $120 \mathrm{~min}$. Predetermined molecular weight standards (Beyotime, Haimen, China) were used as markers. After electrophoresis, the gels were blotted onto a PVDF membrane at $250 \mathrm{~mA}$ for $90 \mathrm{~min}$ at $4^{\circ} \mathrm{C}$. The membrane was blocked in Tris-buffered saline containing $0.1 \%$ Tween 20 (TBST) and 5\% (w/v) non-fat milk for $1 \mathrm{~h}$ at room temperature, washed three times with TBST for $5 \mathrm{~min}$ each. Then the membrane was incubated with the primary monoclonal antibody to P-gp in diluent buffer overnight at $4^{\circ} \mathrm{C}$ (1:200 dilution). After the membrane had been washed with TBST, it was incubated in the appropriate HRP-conjugated anti-mouse secondary antibody (1:2000 dilution) at room temperature for another $1 \mathrm{~h}$ and washed again three times in TBST. The membrane was processed with enhanced chemiluminescence (ECL) detection reagents. The processed membrane was then exposed to photographic films for visualization of the signal. The $\beta$-actin levels were determined by Western blotting to control for equal loading of the lanes.

LC-ESI-MS/MS to assay the Danshensu concentrations in the brain and plasma. LC-ESI-MS/MS was performed according to the method reported in our previous study (10). An Agilent 1100 series HPLC system (Agilent, USA) and a TSQ Quantum Access tandem mass spectrometer (Thermo Electron Corporation, USA) were used to analyze the Danshensu concentrations in the brain and plasma. The chromatographic conditions and mass spectrum conditions were unified with our previous study (10).

Data analysis. Data were analyzed using SPSS 11.5 statistical software (SPSS Inc., Chicago, IL, USA) and are expressed as the means \pm SEM. One-way ANOVA was used to analyze the significant differences within the different groups. The comparison between two groups was determined by the Student's 

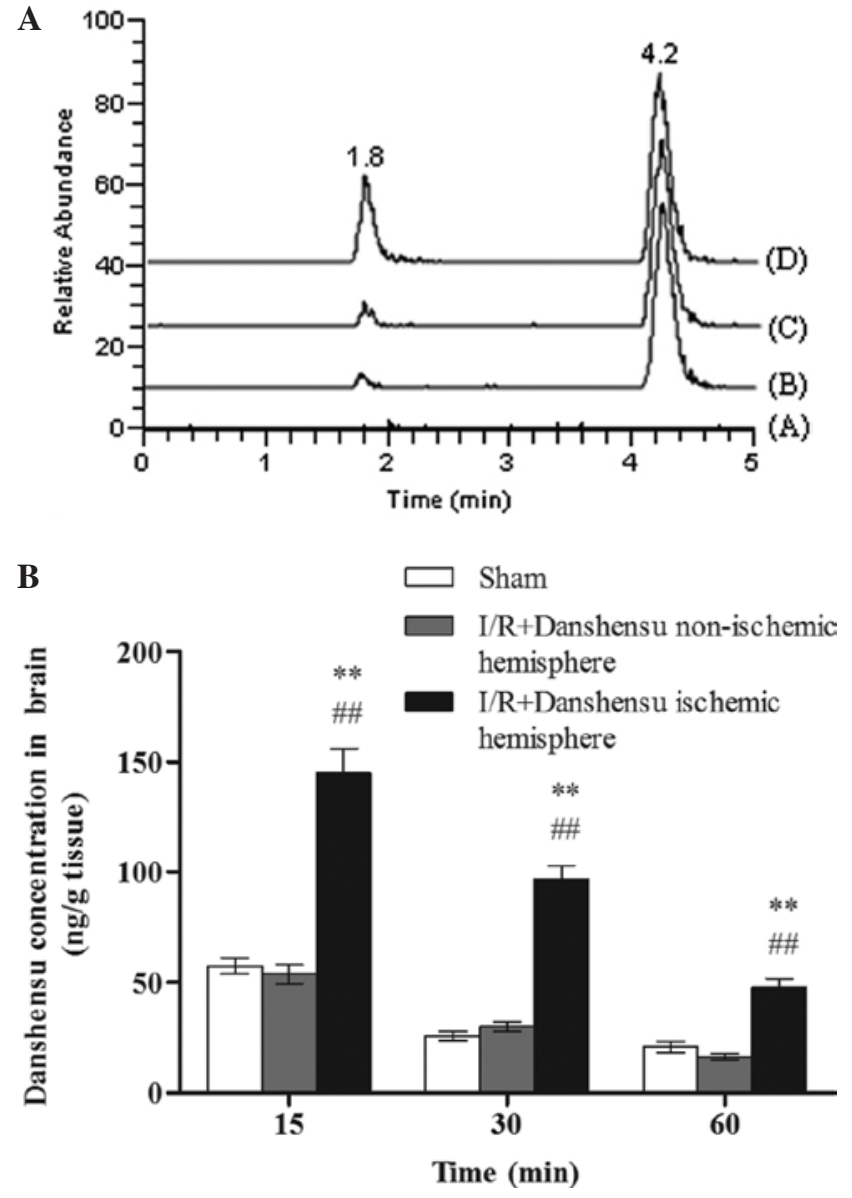

Figure 1. Cerebral ischemia/reperfusion increased the Danshensu concentration in the brain. (A) Selective reactions monitoring (SRM) chromatogram of Danshensu: A, Danshensu and naproxen (internal standard) in the blank rat brain; B, left brain of the sham group spiked with naproxen, $\mathrm{C}$, non-ischemic and $\mathrm{D}$, ischemic hemisphere of the $\mathrm{I} / \mathrm{R}+$ Danshensu group spiked with naproxen. Retention times of Danshensu and naproxen were 1.8 and $4.2 \mathrm{~min}$, respectively. (B) Danshensu concentrations in the ischemic hemisphere of the $\mathrm{I} / \mathrm{R}+$ Danshensu group increased significantly compared with those in the non-ischemic hemisphere of $\mathrm{I} / \mathrm{R}+$ Danshensu, and in the brain of the sham group. ${ }^{* *} \mathrm{P}<0.01$ vs. the non-ischemic hemisphere in the $\mathrm{I} / \mathrm{R}+$ Danshensu group; ${ }^{\# \#} \mathrm{P}<0.01$ vs the sham group.

unpaired t-test. A value of $\mathrm{P}<0.05$ was accepted as indicating a statistically significant difference between the groups.

\section{Results}

Effect of cerebral ischemia/reperfusion on Danshensu concentrations in the brain. The selective reactions monitoring (SRM) chromatogram of Danshensu is shown in Fig. 1A. As shown in Fig. 1B, at 15, 30 and 60 min after cerebral ischemia/ reperfusion, Danshensu concentrations in the ischemic hemisphere of the I/R + Danshensu group increased significantly when compared to those in the non-ischemic hemisphere of the I/R + Danshensu group and to the brain of the sham group $(\mathrm{P}<0.01)$. There was no difference observed in Danshensu concentration between the non-ischemic hemisphere of the $\mathrm{I} / \mathrm{R}+$ Danshensu and in the brain of the sham group $(\mathrm{P}>0.05)$.

Effect of cerebral ischemia/reperfusion on Danshensu concentrations in the plasma. The chromatographic and mass

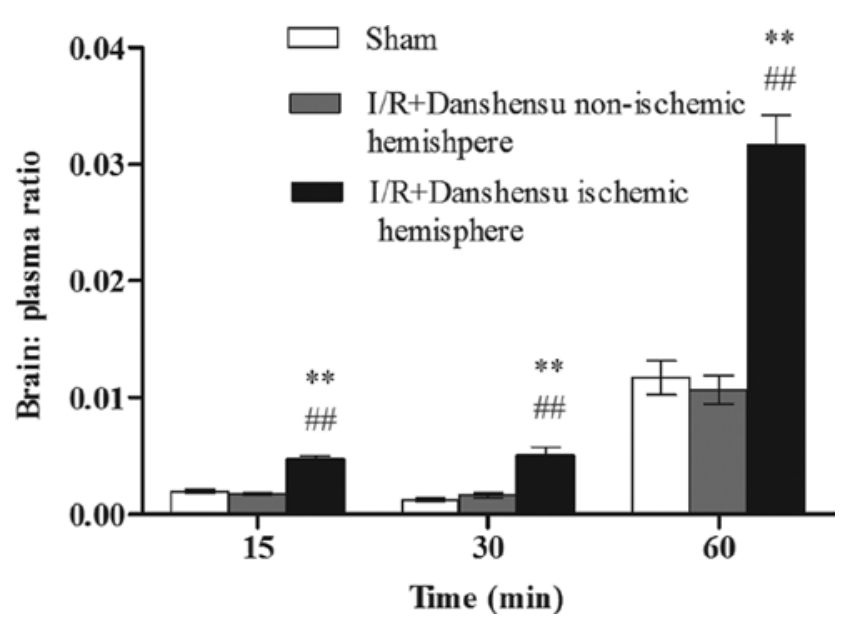

Figure 2. Cerebral ischemia/reperfusion increased the brain:plasma ratios of Danshensu concentrations. Compared with the non-ischemic hemisphere of $\mathrm{I} / \mathrm{R}+$ Danshensu and the brain of the sham group, the brain:plasma ratios of Danshensu concentrations in the ischemic hemisphere of the $\mathrm{I} / \mathrm{R}+$ Danshensu group increased. ${ }^{* *} \mathrm{P}<0.01$ vs. the non-ischemic hemisphere in the $\mathrm{I} / \mathrm{R}+$ Danshensu group; ${ }^{\# \#} \mathrm{P}<0.01$ vs. the sham group.

spectrum conditions were unified with those in the analysis of Danshensu concentrations in the brain. In addition, the retention times of Danshensu and naproxen were 1.7 and $4.3 \mathrm{~min}$, respectively. At 15, 30 and $60 \mathrm{~min}$ after cerebral ischemia/reperfusion, there was no significant difference observed in Danshensu concentrations in the I/R + Danshensu group in plasma $(30755.0 \pm 2950.4,20452.5 \pm 5164.2$ and $1552.75 \pm 280.2 \mathrm{ng} / \mathrm{ml}$, respectively) when compared to those in the sham group $(29616.2 \pm 4272.0,21941.5 \pm 4161.1$ and $1820.0 \pm 329.0 \mathrm{ng} / \mathrm{ml}$, respectively, $\mathrm{P}>0.05)$.

Effect of cerebral ischemia/reperfusion on the brain:plasma ratios of Danshensu concentrations. Compared with the nonischemic hemisphere of the $\mathrm{I} / \mathrm{R}+$ Danshensu and the brain of the sham group, the brain:plasma ratios of Danshensu concentrations in the ischemic hemisphere of $\mathrm{I} / \mathrm{R}+$ Danshensu group significantly increased ( $\mathrm{P}<0.01$, Fig. 2$)$.

Effect of cerebral ischemia/reperfusion and Danshensu on $P$-gp expression in the brain. In the sham group, Danshensu had no effect on the expression of P-gp. P-gp expression in the brain decreased after cerebral ischemia/reperfusion. P-gp expression in the ischemic hemisphere of $I / R$ and $I / R+$ Danshensu groups was lower compared to that in the brain of the sham group. Furthermore, at $60 \mathrm{~min}$ after reperfusion, the P-gp level in the ischemic hemisphere of the I/R + Danshensu group significantly decreased compared to the ischemic hemisphere of the I/R group ( $\mathrm{P}<0.01$, Fig. 3).

\section{Discussion}

Ischemic stroke still leads to long-term disability or even death resulting in a high worldwide socio-economic burden. During the past decades of intensive research, numerous preclinical studies have yielded promising neuroprotective drugs, however, none of these have achieved successful translation into human treatment. 

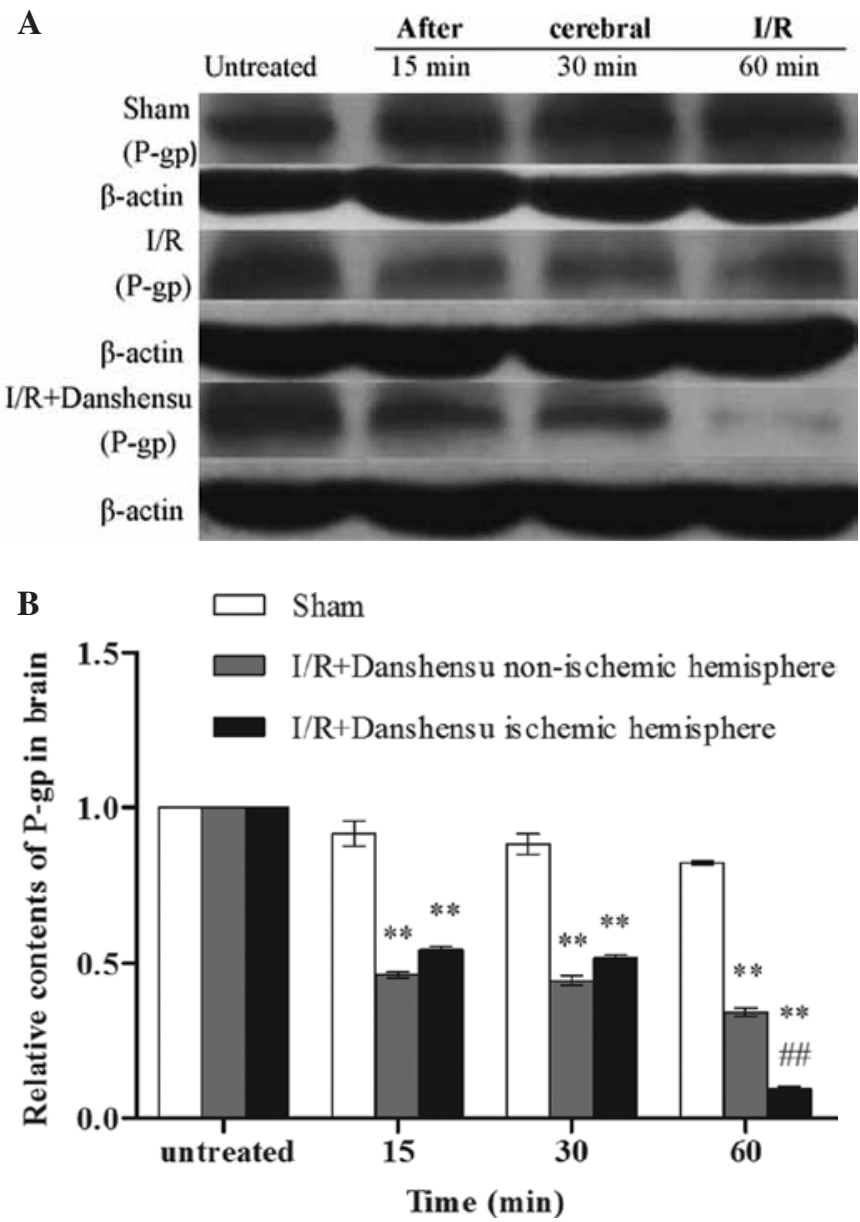

Figure 3. (A) P-gp expression in the brain decreased after cerebral ischemia/ reperfusion. P-gp expression levels in the ischemic hemisphere of the $I / R$ and $\mathrm{I} / \mathrm{R}+$ Danshensu groups were lower compared to those of the sham group. (B) Furthermore, 60 min after reperfusion, $\mathrm{P}$-gp level in the ischemic hemisphere of the I/R + Danshensu group significantly decreased compared to the I/R group. ${ }^{* *} \mathrm{P}<0.01$ vs. the sham group; ${ }^{\# \#} \mathrm{P}<0.01$ vs. the $\mathrm{I} / \mathrm{R}$ group.

Danshen, a traditional Chinese herbal medicine, is the dried root of the plant Salvia miltiorrhiza Bunge belonging to the Labiata family. Danshen contains the lipid-soluble tanshinone I, tanshinone IIA, cryptotanshinone and dihydrotanshinone as well as the water-soluble Danshensu and salvianolic acid B. Studies have shown that tanshinone IIA and tanshinone IIB led to the reduction in brain infarct volume and restoration of neurological function in an experimental model of stroke in mice (12). A recent report demonstrated that tanshinone IIA was effective for attenuating the extent of brain edema formation induced by cerebral ischemia injury in rats (13). Wang et al also reported that tanshinone IIA alleviated the brain damage caused by cerebral ischemia and the protective effect may be through the down-regulation of HMGB1, RAGE, TLR4 and NF- $\mathrm{kB}$ and the up-regulation of claudin-5 expression (14). It was also reported that Danshensu ameliorates the neurological deficit in cerebral ischemia/reperfusion rats (9). Nevertheless, no experiment has focused on the evaluation of tanshinone IIA and Danshensu concentrations in the ischemic hemisphere after cerebral ischemia. In addition, tanshinone I (15), tanshinone IIA (16) and cryptotanshinone (17) were found to be the substrates of P-gp. Previous results from our labora- tory also demonstrated that Danshensu concentrations in the brain of normal rats pretreated with verapamil (P-gp inhibitor) significantly increased (10).

Our results showed that the concentrations of Danshensu in the ischemic hemisphere of the I/R + Danshensu group were higher compared to that in the non-ischemic hemisphere of the $\mathrm{I} / \mathrm{R}+$ Danshensu and the brain of sham group. Furthermore, the brain:plasma ratios of Danshensu concentrations in the ischemic hemisphere of the $\mathrm{I} / \mathrm{R}+$ Danshensu group also increased while there was no difference observed in Danshensu concentrations in the plasma between the I/R + Danshensu and sham groups. These results suggest that it is the transport of Danshensu, instead of the plasma concentration, that affects the concentrations of Danshensu in the brain.

The major challenge for the pharmacotherapy of cerebral ischemia is the delivery of drugs into the brain. The efflux transporters at the BBB play an important role in the restriction of drug entry into the brain. The critical one among these transporters is P-gp which handles a markedly wide range of substrates such as calcium channel blockers, statins, opioids, chemotherapeutic agents, antibiotics and immunosuppressive agents (18). This broad substrate spectrum explains the ability of P-gp to provide cross-resistance to multiple classes of agents for the treatment of cerebral ischemia. Therefore, modulating P-gp is a promising strategy to improve the pharmacotherapy of cerebral ischemia (2).

It is clear that both transporter activity and expression could be modulated by a number of physiological and pathological signals which then lead to either an increase or a decrease in P-gp transport activity. However, to date, the effects of cerebral ischemia on the expression of P-gp are still uncertain (4-7). The present findings showed that the expression of P-gp in the ischemic hemisphere significantly decreased at 15,30 and $60 \mathrm{~min}$ after cerebral ischemia/reperfusion. These disparate results may be caused by the differences in the models chosen, the stress applied and the regions of the brain assayed. Certainly, due to the critical role of P-gp in limiting access of therapeutic drugs into the ischemic hemisphere, it is important to know where, to what extent, and under what circumstances the expression of this BBB transporter is altered after cerebral ischemia.

The concentration of Danshensu in the brain is in line with the results obtained from the Western blot analysis which showed a decline of P-gp expression after cerebral ischemia/ reperfusion. Therefore, the high concentrations of Danshensu in the ischemic hemisphere were partially due to the downregulation of P-gp expression induced by cerebral ischemia/ reperfusion. Furthermore, we found that the expression of P-gp in the ischemic hemisphere of the I/R + Danshensu group significantly decreased compared to the I/R group at $60 \mathrm{~min}$ after Danshensu administration, signifying that the expression of P-gp was down-regulated much more by Danshensu. These results suggest that Danshensu may increase the drug concentration effluxed from the brain by P-gp, and result in synergizing effects. The detailed process of the influence of Danshensu on P-gp expression, particularly the effects of Danshensu on P-gp expression in normal or cerebral ischemia animals, should be investigated in future studies.

In short, our study showed that cerebral ischemia/reperfusion may lead to the increase in Danshensu entry across the BBB 
into the brain, due to the decline of P-gp expression induced by cerebral ischemia/reperfusion, and the further down-regulation of P-gp induced by Danshensu administration.

\section{Acknowledgements}

This study was supported by the Natural Science Foundation of Shandong Province (no. ZR2009CQ004), the Taishan Scholar Project, and the International S \& T Cooperation Program of China (2009DFA31100).

\section{References}

1. Gabathuler R: Blood-brain barrier transport of drugs for the treatment of brain diseases. CNS Neurol Disord Drug Targets 8 : 195-204, 2009

2. Miller DS, Bauer B and Hartz AM: Modulation of P-glycoprotein at the blood-brain barrier: opportunities to improve central nervous system pharmacotherapy. Pharmacol Rev 60: 196-209, 2008.

3. King M, Su W, Chang A,Zuckerman A and Pasternak GW: Transport of opioids from the brain to the periphery by P-glycoprotein peripheral actions of central drugs. Nat Neurosci 4: 268-274, 2001.

4. Felix RA and Barrand MA: P-glycoprotein expression in rat brain endothelial cells: evidence for regulation by transient oxidative stress. J Neurochem 80: 64-72, 2002.

5. Samoto K, Ikezaki K, Yokoyama N and Fukui M: P-glycoprotein expression in brain capillary endothelial cells after focal ischemia in rat. Acta Neurochir Suppl (Wien) 60: 257-260, 1994.

6. Spudich A, Kilic E, Xing H, et al: Inhibition of multidrug resistance transporter-1 facilitates neuroprotective therapies after focal cerebral ischemia. Nat Neurosci 9: 487-488, 2006.

7. Dazert P, Suofu Y, Grube M, et al: Differential regulation of transport proteins in the periinfarct region following reversible middle cerebral artery occlusion in rats. Neuroscience 142 : 1071-1079, 2006
8. Ogura J, Fujikawa A, Maruyama H, Kobayashi M, Itagaki S and Iseki K: Alteration of P-gp expression after intestinal ischemiareperfusion following 16-h fasting in rats. Yakugaku Zasshi 131: 453-462, 2011

9. Yang Y, Xue X and Liu ZP: Protective effect of Danshensu on focal cerebral ischemia-reperfusion injury in rats. Food and Drug 11: 24-26, 2009.

10. Yu PF, Wang WY, Eerdun G, et al: The role of P-glycoprotein in transport of Danshensu across the blood-brain barrier. Evid Based Complement Alternat Med 2011: 713523, 2011.

11. Longa EZ, Weinstein PR, Carlson S and Cummins R: Reversible middle cerebral artery occlusion without craniectomy in rats. Stroke 20: 84-91, 1989.

12. Lam BY, Lo AC, Sun X, Luo HW, Chung SK and Sucher NJ Neuroprotective effects of tanshinones in transient focal cerebral ischemia in mice. Phytomedicine 10: 286-291, 2003.

13. Tang C,XueH,BaiC,Fu R and Wu A: The effects of Tanshinone IIA on blood-brain barrier and brain edema after transient middle cerebral artery occlusion in rats. Phytomedicine 17: 1145-1149, 2010.

14. Wang L, Zhang X, Liu L, et al: Tanshinone II A down-regulates HMGB1, RAGE, TLR4, NF-kappaB expression, ameliorates BBB permeability and endothelial cell function, and protects rat brains against focal ischemia. Brain Res 1321: 143-151, 2010.

15. Li XX, Zhou ZW and Zhou SF: Role of P-glycoprotein in the transport of tanshinone I, one active triterpenoid from Salvia miltiorrhiza. Drug Metab Lett 2: 223-230, 2008.

16. Yu XY, Lin SG, Zhou ZW, et al: Role of P-glycoprotein in the intestinal absorption of tanshinone IIA, a major active ingredient in the root of Salvia miltiorrhiza Bunge. Curr Drug Metab 8: 325-340, 2007.

17. Yu XY, Lin SG, Chen X, et al: Transport of cryptotanshinone, a major active triterpenoid in Salvia miltiorrhiza Bunge widely used in the treatment of stroke and Alzheimer's disease, across the blood-brain barrier. Curr Drug Metab 8: 365-378, 2007.

18. Ford JM and Hait WN: Pharmacologic circumvention of multidrug resistance. Cytotechnology 12: 171-212, 1993. 